\title{
Genetic characterisation of the influenza viruses circulating in Bulgaria during the 2019-2020 winter season
}

\author{
Neli Korsun ${ }^{1}$ (1) $\cdot$ Ivelina Trifonova ${ }^{1} \cdot$ Silvia Voleva ${ }^{1} \cdot$ Iliyana Grigorova ${ }^{1} \cdot$ Svetla Angelova $^{1}$
}

Received: 4 March 2021 / Accepted: 4 June 2021 / Published online: 22 June 2021

(c) The Author(s) 2021

\begin{abstract}
Influenza viruses have a high potential for genetic changes. The objectives of this study were to analyse influenza virus circulation in Bulgaria during the 2019/2020 season, to perform a phylogenetic and molecular analyses of the haemagglutinin (HA) and neuraminidase $(N A)$ sequences of representative influenza strains, and to identify amino acid substitutions compared to the current vaccine strains. Seasonal influenza viruses A(H3N2), A(H1N1)pdm09 and B/Victoria-lineage were detected using a real-time RT-PCR in 323 (23.3\%), 149 (10.7\%) and 138 (9.9\%) out of 1387 patient samples studied, respectively. The $H A$ genes of $\mathrm{A}(\mathrm{H} 3 \mathrm{~N} 2)$ viruses analysed belonged to clades 3C.3a (21 strains) and 3C.2a (5 strains): subclades 3C.2a1b + T131K, 3C.2a1b + T135K-B and 3C.2a1b + T135K-A. The clade 3C.3a and subclade 3C.2a1b viruses carried 5 and 14-17 substitutions in $H A$, as well as 3 and 9 substitutions in $N A$, respectively, in comparison with the A/Kansas/14/2017 vaccine virus, including some substitutions in the $H A$ antigenic sites A, B, C and E. All $21 \mathrm{~A}(\mathrm{H} 1 \mathrm{~N} 1)$ pdm09 viruses sequenced fell into 6B.1A5A subclade. Amino acid sequence analysis revealed the presence of 7-11 substitutions in $H A$, compared to the A/ Brisbane/02/2018 vaccine virus, three of which occurred in antigenic site $\mathrm{Sb}$, along with 6-9 changes at positions in NA. All $10 \mathrm{~B} /$ Victoria-lineage viruses sequenced belonged to clade $1 \mathrm{~A}$ with a triple deletion in $H A l$ (genetic group $1 \mathrm{~A}(\Delta 3) \mathrm{B}$ ) and carried 7 and 3 substitutions in $H A$ and $N A$, respectively, with respect to the $\mathrm{B} /$ Colorado/06/2017 vaccine virus. The results of this study confirm the rapid evolution of influenza viruses and the need for continuous antigenic and genetic surveillance.
\end{abstract}

Keywords Influenza virus · Genetic characterisation · Amino acid substitution

\section{Introduction}

Influenza is one of the most common infectious illnesses in humans that affects annually $5-10 \%$ of the adult population and $20-30 \%$ of children causing a huge number of outpatient visits, hospitalisations and fatal cases, thus imposing a substantial public health burden [1]. Since 2009, two subtypes of influenza $A$ viruses, $A(H 1 N 1) p d m 09$ and $A(H 3 N 2)$, and two genetic lineages of $\mathrm{B}$ viruses, $\mathrm{B} /$ Victoria and $\mathrm{B} /$ Yamagata, have been circulating during seasonal epidemics. There are temporal and geographical variations in their prevalence,

Edited by Hartmut Hengel.

Neli Korsun

neli_korsun@abv.bg

1 Department of Virology, National Laboratory "Influenza and ARI", National Centre of Infectious and Parasitic Diseases, Sofia, Bulgaria clinical activity, as well as in the antigenic and genetic characteristics of the circulating strains.

Influenza viruses belong to the Orthomyxoviridae family and have a segmented, negative-sense, single-stranded RNA genome, each segment of which encodes at least one protein [2]. The surface glycoproteins haemagglutinin $(H A)$ and neuraminidase $(N A)$ form discrete spikes and play an important part in a viral life cycle. $H A$ mediates the binding of the virus to the sialic acids as cellular receptors and the entry of the viral ribonucleoprotein into the cell cytoplasm by fusing of viral envelope and cell membrane. $N A$ destroys sialic acid residues on the cell surface and provides the release of newly formed influenza viruses from the infected cells. $H A$ is the primary target for virus-neutralising antibodies and together with $N A$ is a key component of influenza vaccines.

Vaccination is currently the best way to reduce the influenza morbidity and mortality, but the effectiveness of influenza vaccines is often suboptimal due to the highly variable nature of influenza viruses and their ability to evade the existing humoral immunity. Understanding the molecular 
mechanisms by which influenza viruses avoid the immunity induced by a prior natural infection or vaccination is crucial for the development of effective prevention and control strategies. Due to the absence of a proofreading capability of the viral RNA polymerase complex and the high replication rate of influenza viruses, point mutations occur constantly in the viral genome. Mutations which enable the virus to overcome host immune protection, are accumulated in $H A$ and, to a lesser extent, in $N A$ as a result of immune selection pressure, a process known as antigenic drift [3]. Mutant viruses with altered antigenic characteristics are capable of causing reinfections in immune individuals and repeated epidemics in the general population. It has been found that a limited number of amino acid substitutions at the antibodybinding sites around the receptor-binding pocket can result in escape recognition of neutralising antibodies. With the help of escape mutants, five epitopes of neutralising antibodies, known as antigenic sites, have been identified in the globular heads of the HAs of both $\mathrm{A}(\mathrm{H} 1 \mathrm{~N} 1)(\mathrm{Ca} 1, \mathrm{Ca} 2, \mathrm{Cb}$, $\mathrm{Sa}, \mathrm{Sb}$ ) and $\mathrm{A}(\mathrm{H} 3 \mathrm{~N} 2)$ (A-E) viruses [4, 5]. Influenza type B viruses contain four major immunodominant epitopes in the HA1 subunit comprising the 120-loop, 150-loop, 160-loop, and 190-helix [6]. In addition to mutations, glycosylation of $H A$ and NA (attachment of an oligosaccharide to the sequon $\mathrm{N} \mathrm{X} \mathrm{S/T,} \mathrm{where} \mathrm{X} \mathrm{may} \mathrm{represent} \mathrm{any} \mathrm{amino} \mathrm{acid} \mathrm{except} \mathrm{P}$ ) is another important mechanism used by viruses to evade the antibody-mediated immunity. Recent studies have suggested that the attached glycan chains shield antigenically significant regions reducing access of antibodies and protect the enzymatic sites of $N A$ [7]. The frequent emergence of new genetic variants of influenza viruses that are rapidly replacing previously circulating strains, necessitates regular updates in the influenza vaccines composition in order to match epidemic strains.

Continuous monitoring of circulating influenza viruses is needed to identify new genetic variants of influenza viruses with the potential to avoid host immune defence, with increased virulence, transmissibility or reduced susceptibility to antiviral drugs. The main objectives of this study were to analyse the influenza virus circulation in Bulgaria during the 2019/2020 season and to determine the genetic and molecular characteristics of the detected viruses as compared to the current vaccine strains.

\section{Materials and methods}

\section{Study population and specimen collection}

From October 2019 to March 2020, patients experiencing acute respiratory infection (ARI) from all 28 regions of the country were enrolled in the national influenza surveillance programme (Fig. 1). The diagnosis of each patient was determined by their attending physician based on standard clinical criteria. Nasopharyngeal specimens from the enrolled patients were collected using polyester collection swabs during the visit to the general practitioner or within the first $24 \mathrm{~h}$ of admission. The containers containing a nasopharyngeal swab placed into $2 \mathrm{ml}$ of virus transport medium, were stored at $4{ }^{\circ} \mathrm{C}$ for up to $72 \mathrm{~h}$ and shipped to the National Laboratory "Influenza and ARI", which is recognised by the World Health Organization (WHO) as a National Influenza Centre. Specimens were processed immediately or stored at $-80{ }^{\circ} \mathrm{C}$ before testing.

\section{Extraction of viral nucleic acids and real-time RT-PCR}

Viral RNAs were extracted automatically from nasopharyngeal specimens using a commercial ExiPrep Dx Viral DNA/ RNA kit and ExiPrep 16DX equipment (BioNeer, Korea) in accordance with the manufacturer's instructions. Clinical specimens were screened for influenza viruses by a real-time RT-PCR method using a SuperScript III Platinum ${ }^{\circledR}$ OneStep qRT-PCR System (Invitrogen, USA). Primers, probes and positive controls were provided by the International Reagent Resource, USA. Amplification was performed with a CFX96 thermal cycler (Bio-Rad) in accordance with the protocol recommended by the Centers for Disease Control and Prevention (CDC), Atlanta, USA (reverse transcription at $50{ }^{\circ} \mathrm{C}$ for $30 \mathrm{~min}$, Taq activation at $95{ }^{\circ} \mathrm{C}$ for $2 \mathrm{~min}$, followed by 45 cycles of denaturation at $95{ }^{\circ} \mathrm{C}$ for $15 \mathrm{~s}$ and annealing at $55^{\circ} \mathrm{C}$ for $30 \mathrm{~s}$ ) [8].

\section{Viral isolation and antigenic characterisation}

PCR-positive clinical specimens with a high viral load $\left(C_{t}\right.$ value $<28$ ) were selected and inoculated onto cultures of Madin Darby canine kidney (MDCK) and MDCK-SIAT1 (a cell line expressing increased levels of sialyl- $\alpha 2,6$ galactose moieties [9]). Cultures were incubated at $35^{\circ} \mathrm{C}$ in an atmosphere of $5 \% \mathrm{CO}_{2}$ and observed daily for 7 days for visual cytopathic effect. The presence of virus in culture was confirmed by haemagglutination assay following standard protocols using a $1 \%$ suspension of guinea pig red blood cells. Antigenic characterisation of influenza isolates was performed by the haemagglutination inhibition (HAI) assay following the WHO Manual, using vaccine viruses/ antigens and their corresponding antisera kindly provided by the WHO Collaborating Centres (WHO-CCs) in London and CDC, Atlanta [10]. More detailed HAI assays of representative Bulgarian influenza isolates with panels of reference viruses and antisera were performed at the WHO-CCs in London and Atlanta. Viral isolates were considered as antigenically related to the vaccine virus if they showed no more than a fourfold reduced HAI titre with antiserum raised 


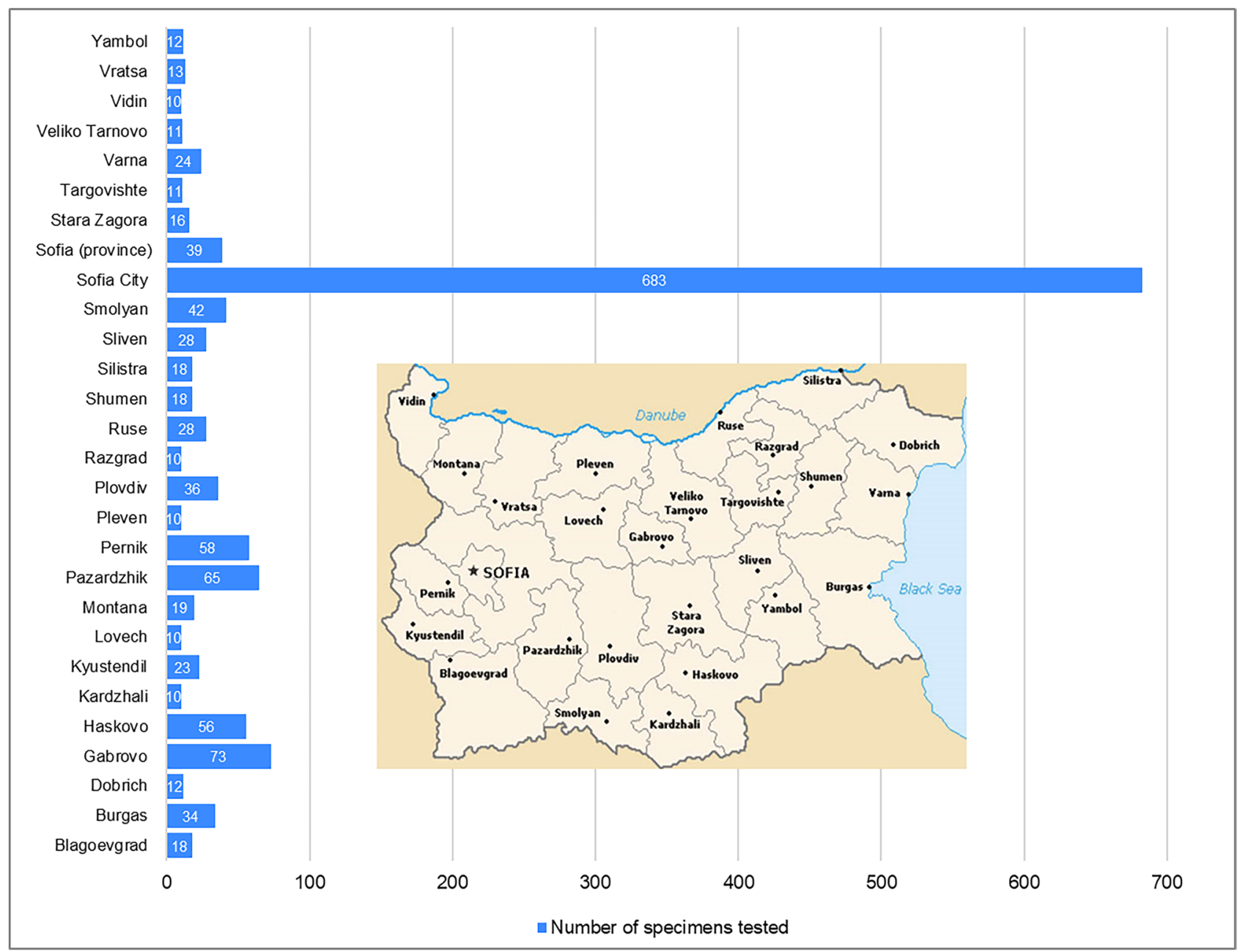

Fig. 1 Number of specimens taken for influenza virus testing in all 28 regions of Bulgaria, 2019/2020 season

against the vaccine virus, as compared to the homologous titre. A reduction of at least eightfold in the HAI titres was considered a signal of antigenic difference.

\section{Genetic characterisation}

The sequencing of influenza viruses detected in Bulgaria during the 2019/2020 season was performed at the WHOCCs in London and Atlanta. Consensus sequences have been deposited in the EpiFlu database of the Global Initiative on Sharing All Influenza Data (GISAID) [11]. For phylogenetic analyses, sequences of study viruses, reference viruses with known genetic group identities determined by the WHOCCs [12], vaccine strains, and viruses representing different countries of Europe during the 2019/2020 season, were retrieved from the EpiFlu database of GISAID (Supplementary Table). Multiple alignments for $H A$ and $N A$ sequences were performed using the MUSCLE algorithm embedded in the MEGA, version 6.06 software [13]. The best-fit nucleotide substitution models for phylogenetic analysis of $H A$ (Hasegawa-Kishino-Yano model with a gamma distribution, $\mathrm{HKY}+\mathrm{G}$ ) and $N A$ (Tamura 3-parameter model with gamma distribution, $\mathrm{T} 92+\mathrm{G}$ ) were determined using MEGA 6.06. Phylogenetic trees were constructed using Maximum Likelihood method. $H A$ and $N A$ nucleotide sequences of the Bulgarian influenza viruses analysed in this study are available in GISAID under the identifiers presented in the Supplementary Table.

\section{Deduced amino acid sequence analysis and prediction of $\mathrm{N}$-glycosylation motifs}

Deduced amino acid sequences were generated by translating nucleotide sequences with the standard genetic code using MEGA 6.06 software. The amino acid sequence identities were calculated using FluServer [14]. The presence of putative $N$-linked glycosylation sites, N X S/T (sequon), 
where $\mathrm{X}$ can be any amino acid except proline, was identified using the NetNGlyc 1.0 Server [15].

\section{Statistics}

The detection rates of the individual viruses were compared using Chi-square or Fisher's exact tests for categorical variables. $P$ values $<0.05$ were considered statistically significant.

\section{Results}

The 2019/2020 influenza epidemic lasted for 6 weeks (weeks 2-8 of 2020) and peaked at week 5. A second, small peak in morbidity due to B/Victoria-lineage viruses was registered at week 10 of 2020 (Fig. 2).

\section{Patient characteristics}

A total of 1387 patients presenting with ARI were tested for influenza viruses. About 23.3\% (323/1387) of these patients attended outpatient healthcare centres, 76.7\% (1064/1387) were hospitalised patients, of which 27 were treated in intensive care units (ICUs). The patients' ages ranged from
13 days to 88 years; 676 (48.7\%) of the participants were male. Most clinical samples $(683,49.2 \%)$ were obtained from patients living in the capital Sofia. The number of samples tested from other regions ranged from 10 to 73 (Fig. 1).

\section{Influenza virus detection}

Influenza viruses were identified in 610 (44\%) study subjects: 138 (42.7\%) outpatients and 472 (44.4\%) inpatients. Influenza type A and type B represented 77.4\% (472/610) and $22.6 \%(138 / 610)$ of all detected influenza viruses, respectively. Of the influenza A cases, $323(68.4 \%)$ were subtyped as $\mathrm{A}(\mathrm{H} 3 \mathrm{~N} 2)$ and $149(31.6 \%)$ were subtyped as $\mathrm{A}(\mathrm{H} 1 \mathrm{~N} 1) \mathrm{pdm} 09$. All 138 (22.6\%) type B viruses were determined to be $\mathrm{B} /$ Victoria-lineage. The proportions of influenza $\mathrm{A}(\mathrm{H} 3 \mathrm{~N} 2), \mathrm{A}(\mathrm{H} 1 \mathrm{~N} 1) \mathrm{pdm} 09$ and B/Victoria-lineage virus infections among outpatients were $24.5 \%$ (79/323), 9.9\% (32/323), and $8.4 \%$ (27/323), respectively, while among the inpatients these proportions were as follows: $22.9 \%$ (244/1064), 11\% (117/1064), and 10.4\% (111/1064), respectively, without statistically significant differences. The percentage of detected $\mathrm{A}(\mathrm{H} 1 \mathrm{~N} 1) \mathrm{pdm} 09$ viruses in the capital Sofia was similar to that in other regions of the country ( $10 \%$ vs $11.5 \%)$, while the percentage of detected $\mathrm{A}(\mathrm{H} 3 \mathrm{~N} 2)$ viruses was higher in the regions outside Sofia (26.3\% vs

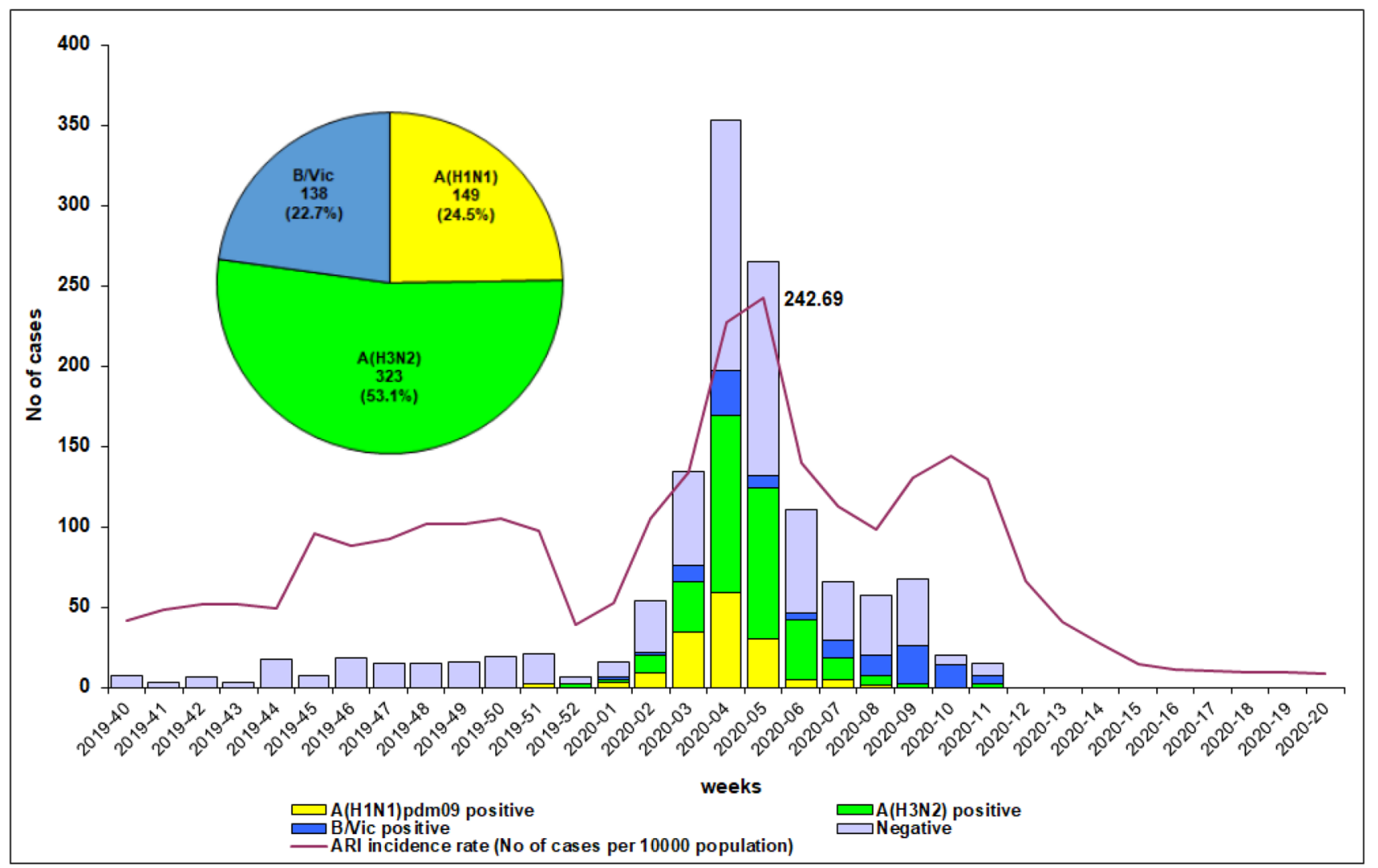

Fig. 2 Weekly distribution of patients tested for influenza viruses in Bulgaria, season 2019/2020 
$20.2 \%, P<0.05)$, and the percentage of detected B/Victoria-lineage viruses was higher in Sofia city (13\% vs $6.7 \%$, $P<0.05)$.

The first influenza detection, an $\mathrm{A}(\mathrm{H} 1 \mathrm{~N} 1) \mathrm{pdm} 09$ virus, occurred in week 51/2019, and the last, an $\mathrm{A}(\mathrm{H} 3 \mathrm{~N} 2)$ virus, occured in week 11/2020. Increased circulation of influenza B/Victoria-lineage viruses was seen at weeks 8-10 of 2020 (Fig. 2). After week 11/2020, influenza virus testing was discontinued due to overloading of the laboratory with SARS-CoV-2 testing.

\section{Phylogenetic and antigenic characterisation}

A total of 57 influenza viruses detected in 13 clinical specimens and 70 cell-culture isolates, were sequenced at WHO$\mathrm{CCs}$ in London and Atlanta and deposited in the EpiFlu database of GISAID. Phylogenetic analysis was performed to determine the affiliation of Bulgarian strains to the genetic groups of globally circulating influenza viruses. The antigenic characterisation using HAI assays was aimed to determine the antigenic similarity of the Bulgarian isolates with the current vaccine strains.

The $H A$ genes of $26 \mathrm{~A}(\mathrm{H} 3 \mathrm{~N} 2)$ viruses sequenced fell into genetic clade 3 C. $3 \mathrm{a}(21 / 26,80.8 \%)$ and clade 3 C. $2 \mathrm{a}$, subclades 3C. $2 \mathrm{a} 1 \mathrm{~b}+\mathrm{T} 131 \mathrm{~K}(1 / 26,3.8 \%), 3 \mathrm{C} .2 \mathrm{a} 1 \mathrm{~b}+\mathrm{T} 135 \mathrm{~K}-$ B $(3 / 26,11.5 \%)$ and 3 C. $2 \mathrm{a} 1 \mathrm{~b}+\mathrm{T} 135 \mathrm{~K}-\mathrm{A}(1 / 26,3.8 \%)$ (Fig. 3). The eight clade 3C.3a viruses and one subclade $3 \mathrm{C} .2 \mathrm{a} 1 \mathrm{~b}+\mathrm{T} 135 \mathrm{~K}-\mathrm{A}$ virus analysed by HAI assay were inhibited by the post-infection ferret antisera raised against the egg-propagated northern hemisphere (NH) 2019/2020 vaccine virus $\mathrm{A} / \mathrm{Kansas} / 14 / 2017$ (clade 3C.3a) at titres 2- to 4-fold lower than the homologous titre of the antiserum. The three subclade 3 C. $2 \mathrm{a} 1 \mathrm{~b}+\mathrm{T} 135 \mathrm{~K}-\mathrm{B}$ viruses analysed by HAI assay exhibited $>$ fourfold titre reductions compared to the vaccine virus and were antigenically distinct.

Phylogenetic analysis of the $H A$ genes of all $21 \mathrm{~A}(\mathrm{H} 1 \mathrm{~N} 1)$ pdm09 viruses sequenced showed that they clustered within the subclade 6B.1A5A (Fig. 4). Ferret antisera raised against egg-propagated vaccine virus A/Brisbane/02/2018 inhibited the eleven antigenically characterised $\mathrm{A}(\mathrm{H} 1 \mathrm{~N} 1)$ pdm09 viruses at titres equal to, or 2- to 4-fold lower than the homologous titre of the antiserum.

All the $10 \mathrm{~B} /$ Victoria-lineage viruses belonged to $H A$ clade $1 \mathrm{~A}$ with a three amino acid deletion in $H A l$ (positions 162-164) (Fig. 5). The nine viruses analysed by HAI assay were poorly recognised by antisera raised against egg-propagated vaccine virus, B/Colorado/06/2017, which contains a two amino acid deletion in $H A$ (positions 162 and 163).

\section{HA and NA protein sequence analysis}

$H A$ and NA protein sequences of influenza viruses circulating in Bulgaria during the 2019/2020 season were compared to those of egg-propagated vaccine strains in order to identify substitutions that could affect vaccine effectiveness.

\section{$A(\mathrm{H} 3 \mathrm{~N} 2)$}

The comparison of the $H A$ amino acid sequences of 26 Bulgarian A(H3N2) strains with the NH 2019/2020 vaccine strain, A/Kansas/14/2017 (clade 3C.3a), revealed a similarity ranged from 98.763 to $99.293 \%$ for clade 3 C.3a viruses and from 95.583 to $96.643 \%$ for subclade 3 C. $2 \mathrm{a} 1 \mathrm{~b}$ viruses [14]. HAs of Bulgarian clade 3C.3a viruses differed from that of vaccine virus with substitutions N190D (all 21 strains), K264E $(n=5)$ and $\mathrm{K} 276 \mathrm{R}(n=11)$ in the $H A l$ domain; and M17L $(n=21)$ and A201V $(n=21)$ in the HA2 domain. Viruses in subclades 3C.2a1b had more changes compared to the vaccine virus: $\mathrm{E} 50 \mathrm{~K}(n=3), \mathrm{E} 62 \mathrm{G}(n=5)$, N91S $(n=5), \mathrm{K} 92 \mathrm{R}(n=5), \mathrm{N} 121 \mathrm{~K}(n=5), \mathrm{T} 135 \mathrm{~K}(n=4)$, S137F ( $n=3), \mathrm{K} 144 \mathrm{~S}(n=5), \mathrm{S} 159 \mathrm{Y}(n=5), \mathrm{K} 160 \mathrm{~T}(n=5)$ resulting in the acquisition of an $N$-linked glycosylation sequon, N171K $(n=5)$, N190D $(n=4)$ and R326K $(n=5)$ in HA1; I77V $(n=5), \mathrm{M} 149 \mathrm{I}(n=5)$ and G155E $(n=4)$ in HA2. Nine substitutions in subclade $3 \mathrm{C} .2 \mathrm{a} 1 \mathrm{~b}$ viruses were located at the antigenic sites: T135K, S137F and K144S - at site A; S159Y, K160T and N190D_at site B; E50K-at site C; E62G and N91S - at site E. Substitution N190D was located on 190-helix (188-194), which is part of antigenic site B, as well as the receptor-binding site (RBS); S144K was very close to the RBS. Thirteen potential $N$-glycosylation sites in $H A$ (HA1 positions $8,22,38,45,63,122,126,133,158$, $165,246$, and 285 , and $H A 2$ position 154$)$ were identified. Six of them were located at the antigenic epitopes: position 63 (antigenic site E); positions 122, 126 and 133 (antigenic site A); and positions 158 and 246 (antigenic site B).

All the Bulgarian clade $3 \mathrm{C} .3 \mathrm{a}$ viruses shared the $N A$ amino acid substitutions I57M, N161S and P386S when they were compared with the sequence of A/Kansas/14/2017 strain. The five viruses in subclades $3 \mathrm{C} .2 \mathrm{a} 1 \mathrm{~b}$ possessed several NA amino acid substitutions: R75K, P126L, I140L, A149V, H155Y, K220N, V303I, S315R and T329S. None of the $N A$ sequences analysed contained substitutions in the catalytic site residues (R118, D151, R152, R224, E276, R292, R371, and Y406) that directly contact the sialic acid, and in the framework residues (E119, R165, W178, S179, D198, I222, E227, H274, E277, N294, and E425) that support the catalytic site [16]. Eight potential $N$-linked glycosylation sequons (at positions 61, 70, 86, 146, 200, 234, 245 and 367) were identified, two of which (146 and 367) were located around the enzyme active site [17].

\section{A(H1N1)pdm09}

$H A$ amino acid sequence identity of the 21 viruses studied ranged from 97.350 to $98.587 \%$ compared to the vaccine 


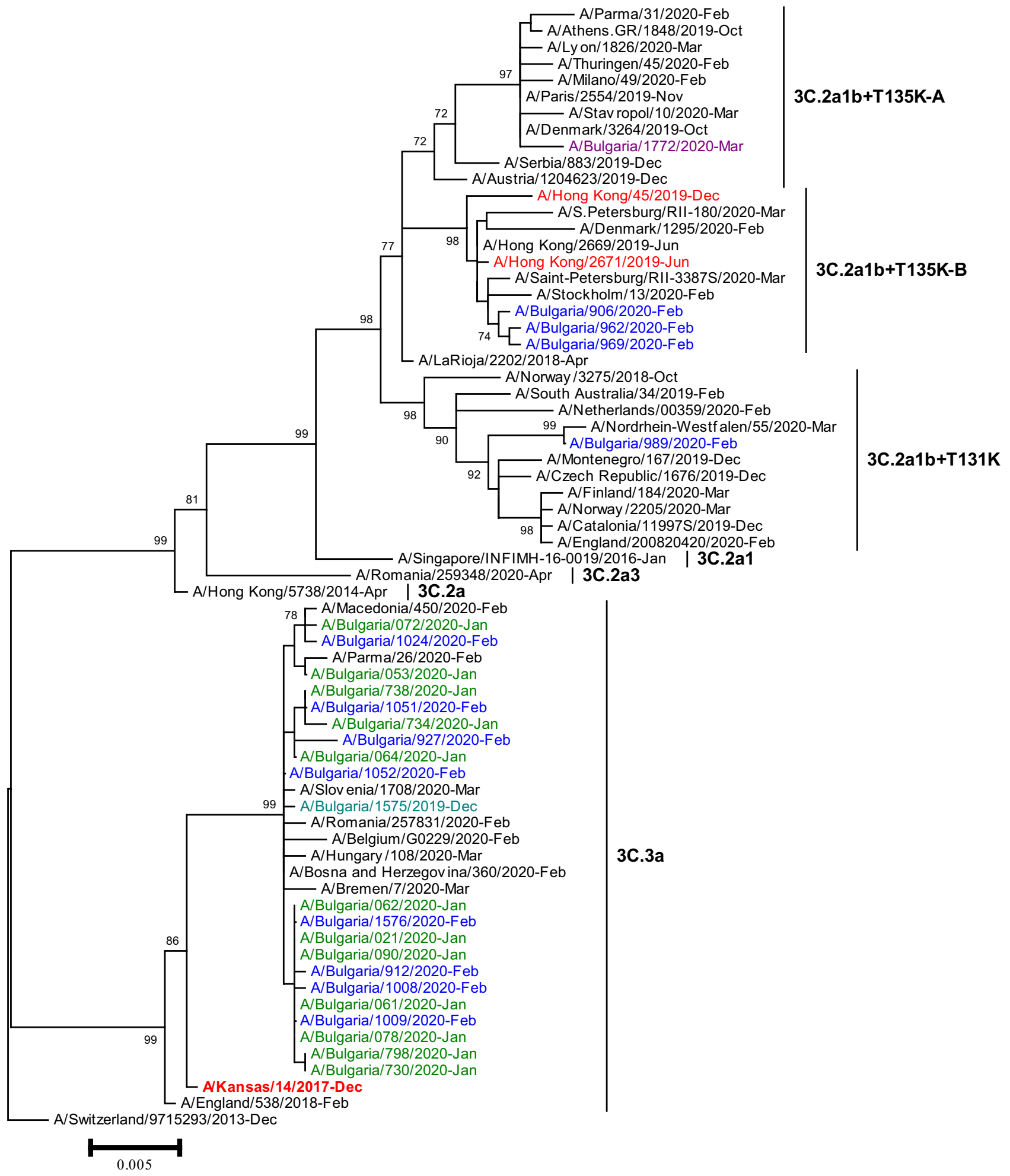

Fig. 3 Phylogenetic analysis of the $H A$ nucleotide sequences from influenza $\mathrm{A}(\mathrm{H} 3 \mathrm{~N} 2)$ viruses detected in Bulgaria during the 2019/2020 season. The tree is rooted at A/Switzerland/9715293/2013. Vaccine viruses A/Kansas/14/2017 (egg-based, 2019-2020 NH influenza season), A/Hong Kong/2671/2019 (egg-based, 2020-2021 NH and 2021 SH influenza seasons) and A/Hong Kong/45/2019 (cell/ recombinant-based, 2020-2021 NH and 2021 SH influenza seasons) are indicated in red. Bulgarian viruses detected from December 2019 to March 2020 are indicated in teal, green, blue and purple, respectively 


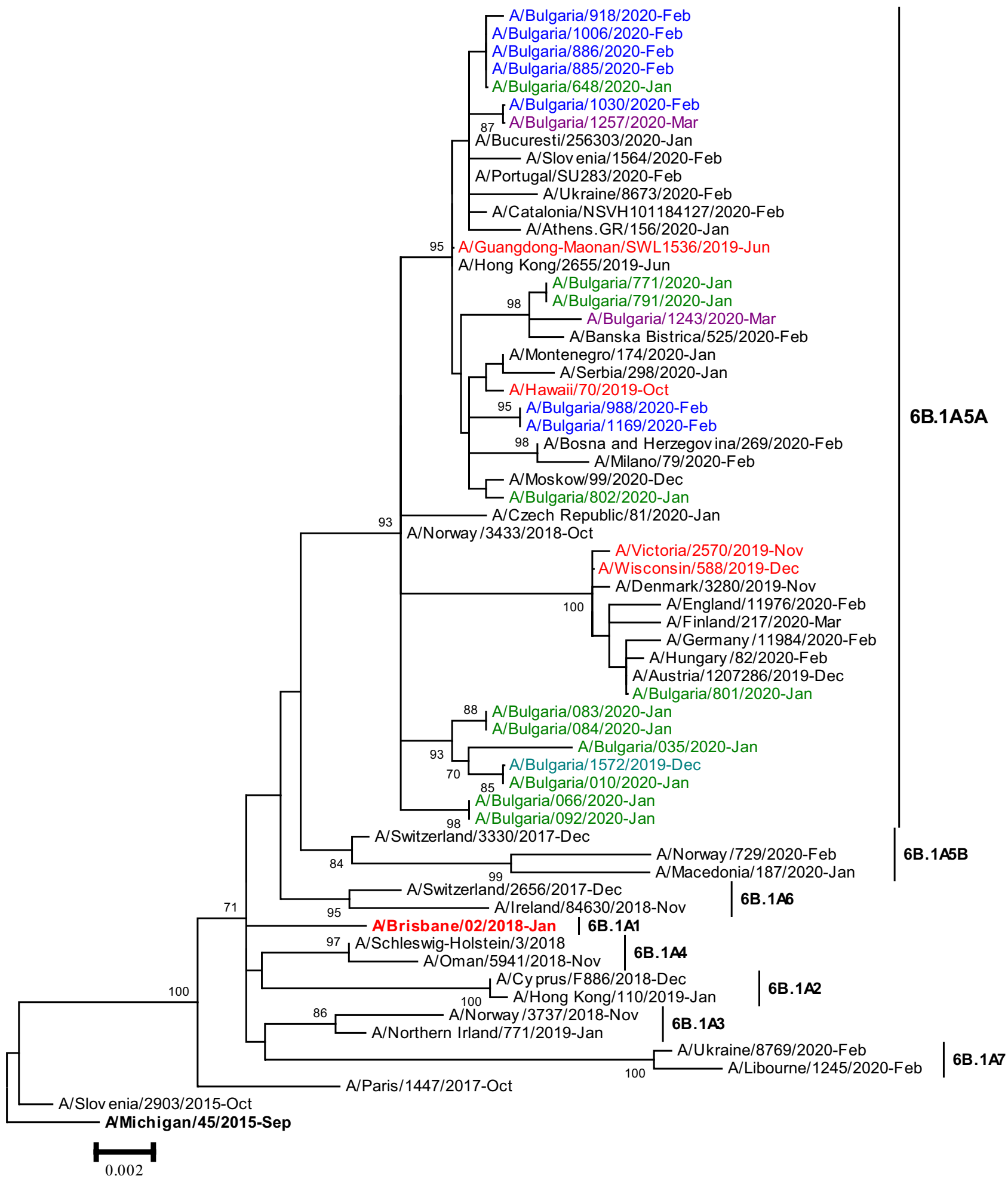

Fig. 4 Phylogenetic analysis of the $H A$ nucleotide sequences from influenza $\mathrm{A}(\mathrm{H} 1 \mathrm{~N} 1) \mathrm{pdm} 09$ viruses detected in Bulgaria during the 2019/2020 season. The tree is rooted at A/Michigan/45/2015. Vaccine viruses A/Brisbane/02/2018 (egg-based, 2019-2020 NH influenza season), A/Guangdong-Maonan/SWL1536/2019 (egg-based, 2020$2021 \mathrm{NH}$ influenza season), A/Hawaii/70/2019 (cell/recombinant- based, 2020-2021 NH influenza season), A/Victoria/2570/2019 (eggbased, $2021 \mathrm{SH}$ influenza season), and A/Wisconsin/588/2019 (cell/ recombinant-based, $2021 \mathrm{SH}$ influenza season), are indicated in red. Bulgarian viruses detected from December 2019 to March 2020 are indicated in teal, green, blue and purple, respectively 


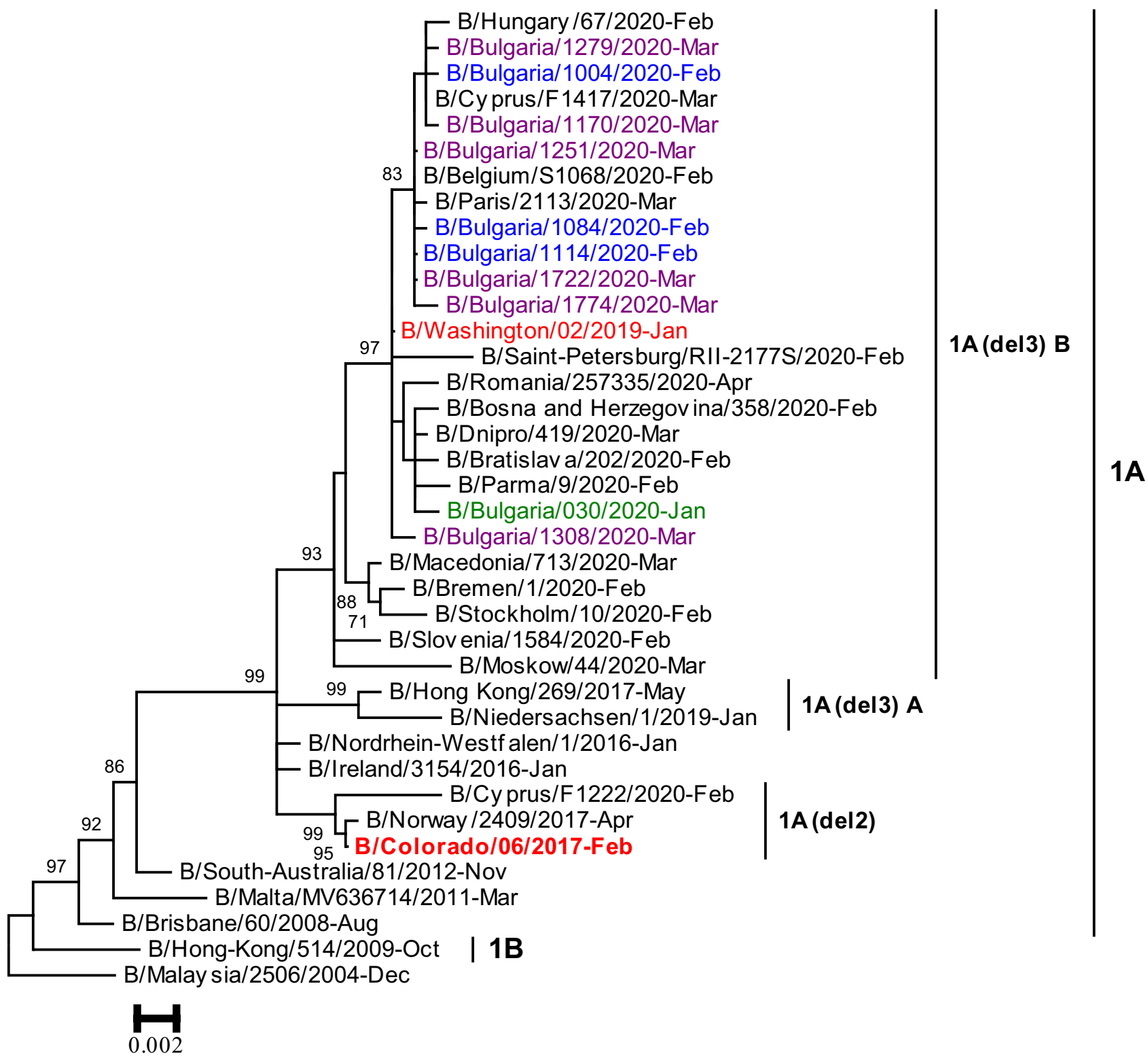

Fig. 5 Phylogenetic analysis of the $H A$ nucleotide sequences from influenza B/Victoria-lineage viruses detected in Bulgaria during the 2019/2020 season. The tree is rooted at B/Malaysia/2506/2004. Vaccine viruses B/Colorado/06/2017 (2019-2020 NH influenza season)

virus, A/Brisbane/02/2018 [14]. All the A(H1N1)pdm09 HA sequences contained 3 amino acid changes in $H A 1, \mathrm{~N} 129 \mathrm{D}$, T185I and N260D, which defined the 6B.1A5A subclade and several additional substitutions: G45R/K, R223Q, A282P and V298I. Thirteen strains fell in subgroup carrying HAl substitutions D187A and Q189E [12].

Three of the identified amino acid substitutions, T185I, D187A and Q189E, were located at antigenic site $\mathrm{Sb}$. The strain A/Bulgaria/801/2020 fell in subgroup $6 \mathrm{~B} .1 \mathrm{~A} 5 \mathrm{~A}+156 \mathrm{~K}$ as $\mathrm{A} /$ Victoria/2570/2019, the vaccine virus for the 2021 southern hemisphere (SH) influenza season, and and B/Washington/02/2019 (2020-2021 NH and $2021 \mathrm{SH}$ influenza season) are indicated in red. Bulgarian viruses detected from January to March 2020 are indicated in green, blue and purple, respectively

carried several additional substitutions, two of which were located at antigenic site Sa [18]. Nine conserved potential $N$-glycosylation motifs were encoded by the $H A$ genes of the 21 Bulgarian $\mathrm{A}(\mathrm{H} 1 \mathrm{~N} 1) \mathrm{pdm} 09$ viruses: at $H A 1$ positions 10/11, 23, 87, 162, 276, 287 and $H A 2$ positions 154 and 213. The $N$-glycosylation motif at position 162 was located in the antigenic site Sa.

The 20 available $N A$ sequences from Bulgarian $\mathrm{A}(\mathrm{H} 1 \mathrm{~N} 1)$ pdm09 viruses differed from that of $A / B$ risbane/02/2018 at several amino acid positions: T13I (all 20 strains), I40T $(n=7)$, Q51K $(n=20)$, S52N $(n=13)$ (with the loss of an 
$N$-glycosylation sequon), T72N ( $n=7)$ (with the gain of an $N$-glycosylation sequon), F74S $(n=20)$, I389K $(n=20)$, D416N $(n=20)$ and T452I $(n=20)$. Two amino acid substitutions were located at the $N A$ antigenic sites including the residues 83-143, 156-190, 252-303, 330, 332, 340-345, 368, 370, 387-395, 400, 431-435, and 448-468 [19]. NA catalytic site residues $(118 \mathrm{R}, 119 \mathrm{E}, 151 \mathrm{D}, 152 \mathrm{R}, 179 \mathrm{~W}$, 223I, 225R, 277E, 293R, 368R, and 402Y) and their supporting framework residues (156R, 180S, 228E, 247S, 278E, and $295 \mathrm{~N}$ ) were highly conserved [20]. Eight potential $N$-glycosylation motifs at positions $42,50,58,63,68,88$, 146 and 235 were found.

\section{B/Victoria}

All the 10 sequenced Bulgarian B/Victoria-lineage viruses belonged to clade $1 \mathrm{~A}$ with a triple deletion $(\Delta 162-164)$ in HAl. Compared to the current vaccine virus, B/Colorado/06/2017, there was a $H A$ amino acid similarity between 98.110 and $98.797 \%$ [14]. All Bulgarian strains fell in group encoding the K136E substitution in HAl (genetic group $1 \mathrm{~A}(\Delta 3) \mathrm{B})$. Several other amino acid substitutions were also identified in HA: G129D ( $n=10)$, G133R $(n=10)$, V180I $(n=10), \mathrm{T} 197 \mathrm{~N}(n=10)$ resulting in the gain of a potential $N$-glycosylation site, $\mathrm{N} 233 \mathrm{~K}(n=8)$ resulting in the loss of a potential $N$-glycosylation motif in $H A 1$, and K151R $(n=10)$ in $H A 2$. Three amino acid variations were found in the antigenic 120-loop area (positions 116-137); T197N was identified in the 190-helix area (positions 194-202). Twelve potential $N$-linked glycosylation sites were identified at $H A I$ positions 25, 59, 145, 166, 197, 233, 304 and 333, and $H A 2$ positions $145,171,184$ and $216 . N$-linked glycosylation sites 145, 166 and 197 were located at the antigenic sites: 150loop (positions 141-150), 160-loop (positions 162-167) and 190-helix, respectively [21].

The sequenced Bulgarian B/Victoria-lineage viruses carried three NA amino acid substitutions: S283G $(n=6)$, Q371K $(n=7)$ and A395T $(n=7)$. The eight catalytic residues (R116, D149, R150, R223, E275, R292, R374, and Y409) and their 11 framework residues (E117, R154, W177, S178, D197, I221, E226, H273, E276, N293, E428) within $N A$ were conserved among all 7 viruses studied [22]. Four putative $\mathrm{N}$-glycosylation motifs at positions 56, 64, 144 and 284 were identified.

\section{Discussion}

In the present study, the circulation pattern and genetic characteristics of influenza viruses detected in Bulgaria during the 2019/2020 season were examined. Influenza A(H3N2) viruses prevailed (53.1\%), and $\mathrm{A}(\mathrm{H} 1 \mathrm{~N} 1) \mathrm{pdm} 09$ and $\mathrm{B} / \mathrm{Vic}-$ toria-lineage viruses circulated in similar proportions $(24.5 \%$ and $22.7 \%$, respectively). No B/Yamagata-lineage viruses were identified. Cumulative data for the WHO European region showed that most viruses detected (73\%) were type A with a slight predominance $(56 \%)$ of $\mathrm{A}(\mathrm{H} 1 \mathrm{~N} 1) \mathrm{pdm} 09$ viruses over $\mathrm{A}(\mathrm{H} 3 \mathrm{~N} 2)$ viruses $(44 \%)$, and $27 \%$ were type $\mathrm{B}$, with $98 \%$ of them determined to be B/Victoria-lineage [23].

In Bulgaria, influenza A viruses were predominant for most seasons after the 2009/2010 pandemic, except for the 2012/2013 and 2017/2018 seasons. Strong dominance of influenza A(H3N2) viruses was observed in the 2011/2012, 2014/2015 and 2016/2017 seasons, and increased circulation of A(H1N1)pdm09 viruses was seen during the 2010/2011, 2013/2014, and 2015/2016 epidemics [24, 25].

Among the seasonal influenza viruses, $\mathrm{A}(\mathrm{H} 3 \mathrm{~N} 2)$ have been characterised by the highest rate of evolution and significant genetic diversity [26-28]. Since 2009, seven genetic groups and multiple clades/subclades based on the $H A$ gene have been defined within this subtype. In 2014, two genetically and antigenically divergent genetic clades, 3C.2a and 3C.3a, emerged and continued to circulate widely worldwide in the following seasons [29]. Viruses in clade 3C.2a rapidly became predominant in most geographic areas including in Bulgaria [25, 30]. Over this period, 3C.2a viruses diverged into several subclades: 3C.2a1 (3C.2a1a and 3C.2a1b), 3C.2a2, 3C.2a3, and 3C.2a4 [16]. Unlike the previous four seasons, during the 2019/2020 influenza epidemic in Bulgaria, the majority (80.8\%) circulating $\mathrm{A}(\mathrm{H} 3 \mathrm{~N} 2)$ viruses fell into the genetic clade 3C.3a, and the remainder (19.2\%) belonged to subclade 3C.2alb, which included three subdivisions and was not represented in the vaccine. The clade 3 C.3a viruses were antigenically and genetically close to the vaccine virus, while those of subclade $3 \mathrm{C} .2 \mathrm{a} 1 \mathrm{~b}$ and its subdivisions differed significantly due to the presence of a great number of amino acid mismatches, 6-9 of which were within the $H A$ antigenic sites A, B, C and E (Table 1). Antigenic sites $\mathrm{B}$ and $\mathrm{A}$, located on the top of $\mathrm{HAl}$ around the RBS are main targets of human neutralising antibodies. It has been found that from 1968 to 2003, the major antigenic changes have been caused mainly by single amino acid substitutions which occur at only seven positions in $H A$ (145 in site A and 155, 156, 158, 159, 189, 193 in site B) near the RBS [31]. In our study, substitution S159Y was found in one of these key positions. HAs of $\mathrm{A}(\mathrm{H} 3 \mathrm{~N} 2)$ viruses have been characterised by a high degree of glycosylation, which probably facilitates immune evasion. Previous studies showed that the number of $H A$ glycosylation sequons in $\mathrm{A}(\mathrm{H} 3 \mathrm{~N} 2)$ viruses increases in the years following their introduction into the human population. While the pandemic $\mathrm{A}(\mathrm{H} 3 \mathrm{~N} 2)$ strain Hong Kong/1/68 harboured two N-glycosylation sites at positions 81 and 165 on the head of $H A$, the most recent representatives contained 7 additional $\mathrm{N}$-glycosylation sites on the $H A$ globular head and 5 on the stem region [16]. Six potential $N$-glycosylation motifs were identified at the 
Table 1 Number of positions with amino acid changes compared to the vaccine viruses and number of potential N-linked glycosylation motifs in HA and NA of influenza viruses circulating in Bulgaria during the 2019/2020 season

\begin{tabular}{|c|c|c|c|c|c|c|}
\hline \multirow[t]{3}{*}{ Influenza viruses } & \multirow[t]{3}{*}{ Vaccine strains } & \multicolumn{3}{|c|}{$\begin{array}{l}\text { Number of positions with amino acid changes com- } \\
\text { pared to the vaccine viruses }\end{array}$} & \multicolumn{2}{|c|}{$\begin{array}{l}N \text {-glycosyla- } \\
\text { tion motifs }\end{array}$} \\
\hline & & \multicolumn{2}{|l|}{$H A$} & \multirow[t]{2}{*}{$N A$} & \multirow[t]{2}{*}{$H A$} & \multirow[t]{2}{*}{$N A$} \\
\hline & & Total number & $\begin{array}{l}\text { Positions at anti- } \\
\text { genic regions }\end{array}$ & & & \\
\hline A(H1N1)pdm09 & A/Brisbane/02/2018 & 7 & 1 & 6 & 9 & 8 \\
\hline $\mathrm{A}(\mathrm{H} 1 \mathrm{~N} 1) \mathrm{pdm} 09+\mathrm{D} 187 \mathrm{~A}$ and Q189E & & 9 & 3 & 9 & 9 & 8 \\
\hline $\mathrm{A}(\mathrm{H} 1 \mathrm{~N} 1) \mathrm{pdm} 09+\mathrm{N} 156 \mathrm{~K}$ & & 11 & 3 & 9 & 9 & 8 \\
\hline $\mathrm{A}(\mathrm{H} 3 \mathrm{~N} 2) /$ clade $3 \mathrm{C} .3 \mathrm{a}$ & A/Kansas/14/2017 & 5 & - & 3 & 13 & 8 \\
\hline $\mathrm{A}(\mathrm{H} 3 \mathrm{~N} 2) /$ subclade $3 \mathrm{C} .2 \mathrm{a} 1 \mathrm{~b}$ & & $14-17$ & $6-9$ & 9 & 13 & 8 \\
\hline B/Victoria-lineage & B/Colorado/06/2017 & 7 & 3 & 3 & 12 & 4 \\
\hline
\end{tabular}

antigenic regions of Bulgarian $\mathrm{A}(\mathrm{H} 3 \mathrm{~N} 2)$ viruses. Attachment of oligosaccharide chains to the antigenic sites of $H A$ may alter the antigenicity and virulence of influenza viruses [32]. The glycan at position 158 is located immediately adjacent to the amino acid residues at positions 156,158 and 159 that are responsible for major antigenic cluster transitions.

Since the 2009/2010 pandemic, the A(H1N1)pdm09 virus has evolved continuously and has undergone significant diversification in eight major genetic groups and several clades/subclades based on the $H A$ gene. The appearance of the recent clade 6B.1 viruses at the beginning of the $\mathrm{NH}$ $2015 / 2016$ season was accompanied by many severe and fatal influenza cases in a number of countries, including Bulgaria $[33,34]$. The vast majority of recently globally circulating viruses formed a subclade designated 6B.1A5A and all sequenced Bulgarian $\mathrm{A}(\mathrm{H} 1 \mathrm{~N} 1)$ pdm09 viruses belonged to this subclade. A total of 7-11 amino acid variations (three located at antigenic site $\mathrm{Sb}$ and two at site $\mathrm{Sa}$ ) were identified in $H A$ and 6-9 in NA compared to the vaccine virus, A/ Brisbane/2/2018 (Table 1). Attachment of glycan at position 162 of the antigenic site Sa is likely to affect antibody recognition. Despite these genetic changes, subclade 6B.1A5A viruses remained antigenically similar to the vaccine virus as assessed by the use of post-infection ferret antisera raised against the vaccine virus [12]. However, due to the increasing number of viruses A(H1N1)pdm09+D187A and Q189E, which were antigenically distinguishable from the vaccine virus by panels of post-vaccination human sera from the 2019-2020 NH influenza season, A/Guangdong-Maonan/ SWL1536/2019 virus was recommended as a vaccine component for the 2020/2021 NH influenza season [35].

In Bulgaria, all type B viruses detected in 2019/2020 belonged to the Victoria-lineage. Previously, an increased circulation of B/Victoria-lineage viruses was observed in the country in the 2015-2016 season [33]. Recently circulating viruses with a double $(\Delta 162-163)$ or a triple deletion ( $\triangle 162-164)$ in $H A l$ appeared for the first time during the
2016/2017 season [36]. Seven amino acid substitutions in $H A 1$, four of which were located in the antigenic 120-loop $(n=3)$ and 190-helix $(n=1)$, and 5 substitutions in NA were identified in Bulgarian strains with respect to the current vaccine virus (Table 1). According to previous studies, the 120-loop exhibits a highest degree of variations [37]. The 190-helix is a part of the RBS. Amino acid substitutions within these regions could potentially alter antigenicity and virulence $[22,38,39]$. This statement was confirmed by the results of antigen characterisation, which showed that the Bulgarian $\mathrm{B} /$ Victoria-lineage viruses were antigenically distinct from the contemporary vaccine component.

In conclusion, co-circulation of seasonal influenza viruses $\mathrm{A}(\mathrm{H} 3 \mathrm{~N} 2), \mathrm{A}(\mathrm{H} 1 \mathrm{~N} 1) \mathrm{pdm} 09$ and B/Victoria-lineage was observed during the 2019/2020 season in Bulgaria. The results of this study showed the emergence of new genetic variants, some of which had alterations in antigenicity compared to the current vaccine strains. The study confirms the rapid evolution of influenza viruses and the need for continuous antigenic and genetic surveillance.

Supplementary Information The online version contains supplementary material available at https://doi.org/10.1007/s11262-021-01853-w.

Acknowledgements The authors would like to express their special gratitude to collaborators within WHO-CC, London and the Virus Surveillance and Diagnosis Branch, Influenza Division, CDC, Atlanta, for their efforts in antigenic and genetic characterisation of influenza viruses. We also gratefully acknowledge the contributions of team members in the authors' host institutions, and of the originating and submitting laboratories of sequences deposited in the EpiFlu Database of GISAID that were used in this study.

Author contributions Conceptualisation: NK; Formal analysis and investigation: NK, IT, SV, IG, SA; Writing-original draft preparation: NK; Writing — review and editing: SA. All authors read and approved the final manuscript.

Funding This work was supported by the Ministry of Health in Bulgaria ("National Plan of the Republic of Bulgaria for Influenza 
Pandemic Preparedness") and the National Science Foundation (Project DH 13-15/20.12.2017).

Data availability The datasets analysed during the current study are available in the GISAID database (https://www.gisaid.org/).

\section{Declarations}

Conflict of interest The authors declare they have no financial interests.

Ethical approval This work was performed in line with The Code of Ethics of the World Medical Association (Declaration of Helsinki) for experiments involving humans. The study was approved by the Institutional Review Board/ Institutional Ethics Committee of the National Centre of Infectious and Parasitic Diseases (Number IRB 00006384).

Consent to participate Written informed consent to participate in the study was obtained from all individual participants (or parents/ legal guardians in the case of children under 16) before specimen collection and testing.

Open Access This article is licensed under a Creative Commons Attribution 4.0 International License, which permits use, sharing, adaptation, distribution and reproduction in any medium or format, as long as you give appropriate credit to the original author(s) and the source, provide a link to the Creative Commons licence, and indicate if changes were made. The images or other third party material in this article are included in the article's Creative Commons licence, unless indicated otherwise in a credit line to the material. If material is not included in the article's Creative Commons licence and your intended use is not permitted by statutory regulation or exceeds the permitted use, you will need to obtain permission directly from the copyright holder. To view a copy of this licence, visit http://creativecommons.org/licenses/by/4.0/.

\section{References}

1. World Health Organization (WHO) (2017) Influenza. www.who. int/biologicals/vaccines/influenza/en. Accessed 16 Mar 2018.

2. Medina RA, Garcia-Sastre A (2011) Influenza A viruses: new research developments. Nat Rev Microbiol 9:590-603. https:// doi.org/10.1038/nrmicro2613

3. Webster RG, Laver WG, Air GM, Schild GC (1982) Molecular mechanisms of variation in influenza viruses. Nature 296:115121. https://doi.org/10.1038/296115a0

4. Gerhard W, Yewdell J, Frankel ME, Webster R (1981) Antigenic structure of influenza virus haemagglutinin defined by hybridoma antibodies. Nature 290(5808):713-717. https://doi.org/10.1038/ 290713a0

5. Wiley DC, Wilson IA, Skehel JJ (1981) Structural identification of the antibody-binding sites of Hong Kong influenza haemagglutinin and their involvement in antigenic variation. Nature 289:373-378. https://doi.org/10.1038/289373a0

6. Krystal M, Young JF, Palese P, Wilson IA, Skehel JJ, Wiley DC (1983) Sequential mutations in hemagglutinins of influenza B virus isolates: definition of antigenic domains. Proc Natl Acad Sci USA 80:4527-4531. https://doi.org/10.1073/pnas.80.14.4527

7. Zost SJ, Parkhouse K, Gumina ME, Kim K, Diaz Perez S et al (2017) Contemporary H3N2 influenza viruses have a glycosylation site that alters binding of antibodies elicited by egg-adapted vaccine strains. Proc Natl Acad Sci USA 114(47):12578-12583. https://doi.org/10.1073/pnas.1712377114

8. Shu B, Wu KH, Emery S, Villanueva J, Johnson R, Guthrie E et al (2011) Design and performance of the CDC real-time reverse transcriptase PCR swine flu panel for detection of 2009 A(H1N1) pandemic influenza virus. J Clin Microbiol 49:2614-2619. https:// doi.org/10.1128/JCM.02636-10

9. Matrosovich M, Matrosovich T, Carr J, Roberts NA, Klenk HD (2003) Overexpression of the alpha-2,6-sialyltransferase in MDCK cells increases influenza virus sensitivity to neuraminidase inhibitors. J Virol 77(15):8418-8425. https://doi.org/10.1128/jvi. 77.15.8418-8425.2003

10. World Health Organization (WHO) (2011) Manual for the laboratory diagnosis and virological surveillance of influenza. http:// whqlibdoc.who.int/publications/2011/9789241548090_eng.pdf

11. Global Initiative on Sharing All Influenza Data (GISAID). https://www.gisaid.org/

12. WHO Worldwide Influenza Centre, London (September 2020) Interim Report. Report prepared for the WHO annual consultation on the composition of influenza vaccine for the Southern Hemisphere 2021. https://www.crick.ac.uk/sites/default/files/ 2020-10/Crick_SH2021\%20report_Full\%20seasonal.pdf

13. Molecular Evolutionary Genetics Analysis (MEGA) software. http://www.megasoftware.net/

14. FluSurver. http://flusurver.bii.a-star.edu.sg

15. NetNGlyc 1.0 Server. http://www.cbs.dtu.dk/services/NetNG lyc/

16. Allen JD, Ross TM (2018) H3N2 influenza viruses in humans: Viral mechanisms, evolution, and evaluation. Hum Vaccin Immunother 14(8):1840-1847. https://doi.org/10.1080/21645 515.2018.1462639

17. Fang Q, Gao Y, Chen M, Guo X, Yang X, Yang X, Wei L (2014) Molecular epidemiology and evolution of A(H1N1)pdm09 and H3N2 virus during winter 2012-2013 in Beijing, China. Infect Genet Evol 26:228-240. https://doi.org/10.1016/j.meegid.2014. 05.034

18. Wilson JR, Guo Z, Tzeng W-P, Garten RJ, Xiyan X, Blanchard EG et al (2015) Diverse antigenic site targeting of influenza hemagglutinin in the murine antibody recall response to A(H1N1)pdm09 virus. Virology 485:252-262. https://doi.org/ 10.1016/j.virol.2015.08.004

19. Graham M, Liang B, Van Domselaar G, Bastien N, Beaudoin C, Tyler S, Kaplen B, Landry E, Li Y (2011) Nationwide molecular surveillance of pandemic H1N1 influenza A virus genomes: Canada, 2009. PLoS ONE 6:e16087. https://doi.org/10.1371/ journal.pone.0016087

20. Colman PM, Hoyne PA, Lawrence MC (1993) Sequence and structure alignment of paramyxovirus hemagglutinin-neuraminidase with influenza virus neuraminidase. J Virol 67:29722980. https://doi.org/10.1128/JVI.67.6.2972-2980.1993

21. Wang Q, Cheng F, Lu M, Tian X, Ma J (2008) Crystal structure of unliganded influenza B virus hemagglutinin. J Virol 82(6):3011-3020. https://doi.org/10.1128/JVI.02477-07

22. Rivas MJ, Alegretti M, Cóppola L, Ramas V, Chiparelli H, Goñi N (2020) Epidemiology and genetic variability of circulating influenza B viruses in Uruguay, 2012-2019. Microorganisms 8(4):591. https://doi.org/10.3390/microorganisms8 040591

23. European Centre for Disease Prevention and Control. Influenza virus characterisation-Summary Europe, September 2020. https://www.ecdc.europa.eu/sites/default/files/documents/influ enza-characterisation-report-september-2020.pdf

24. Korsun N, Angelova S, Teodosieva A (2016) Virological surveillance of influenza in four recent post-pandemic seasons (2010/11 
to 2013/14) in Bulgaria. Cent Eur J Public Health 24:180-187. https://doi.org/10.21101/cejph.a4297

25. Korsun N, Angelova S, Trifonova I, Tzotcheva I, Mileva S, Voleva $S$ et al (2018) Predominance of influenza $A(H 3 N 2)$ viruses during the 2016/2017 season in Bulgaria. J Med Microbiol 67(2):228239. https://doi.org/10.1099/jmm.0.000668

26. Bedford T, Suchard MA, Lemey P, Dudas G, Gregory V et al (2014) Integrating influenza antigenic dynamics with molecular evolution. Elife 3:e01914. https://doi.org/10.7554/eLife.01914

27. Rambaut A, Pybus OG, Nelson MI, Viboud C, Taubenberger JK, Holmes EC (2008) The genomic and epidemiological dynamics of human influenza A virus. Nature 453(7195):615-619. https:// doi.org/10.1038/nature06945

28. Andrés C, Peremiquel-Trillas P, Gimferrer L, Piñana M, Codina MG, Rodrigo-Pendás JA et al (2019) Molecular influenza surveillance at a tertiary university hospital during four consecutive seasons (2012-2016) in Catalonia, Spain. Vaccine 37(18):2470 2476. https://doi.org/10.1016/j.vaccine.2019.03.046

29. Broberg E, Snacken R, Adlhoch C, Beaute J, Galinska M, Pereyaslov D et al (2015) Start of the 2014/15 influenza season in Europe: drifted influenza $\mathrm{A}(\mathrm{H} 3 \mathrm{~N} 2)$ viruses circulate as dominant subtype. Euro Surveill 20:21023. https://doi.org/10.2807/15607917.ES2015.20.4.21023

30. Korsun N, Daniels R, Angelova S, Ermetal B, Grigorova I, Voleva $S$ et al (2020) Genetic diversity of influenza A viruses circulating in Bulgaria during the 2018-2019 winter season. J Med Microbiol 69(7):986-998. https://doi.org/10.1099/jmm.0.001198

31. Koel BF, Burke DF, Bestebroer TM, van der Vliet S, Zondag GC, Vervaet $G$ et al (2013) Substitutions near the receptor binding site determine major antigenic change during influenza virus evolution. Science 342(6161):976-979. https://doi.org/10.1126/scien ce. 1244730

32. Schulze IT (1997) Effects of glycosylation on the properties and functions of influenza virus hemagglutinin. J Infect Dis 176(Suppl 1):S24-S28. https://doi.org/10.1086/514170
33. Korsun N, Angelova S, Gregory V, Daniels R, Georgieva I, McCauley J (2017) Antigenic and genetic characterization of influenza viruses circulating in Bulgaria during the 2015/2016 season. Infect Genet Evol 49:241-250. https://doi.org/10.1016/j. meegid.2017.01.027

34. Ilyicheva T, Durymanov A, Susloparov I, Kolosova N, Goncharova N, Svyatchenko S et al (2016) Fatal Cases of Seasonal Influenza in Russia in 2015-2016. PLoS ONE 11(10):e0165332. https://doi.org/10.1371/journal.pone.0165332

35. World Health Organization (WHO) (2020) Recommended composition of influenza virus vaccines for use in the 2020-2021 northern hemisphere influenza season

36. Blanton L, Alabi N, Mustaquim D, Taylor C, Kniss K, Kramer N et al (2017) Update: influenza activity in the United States during the 2016-17 season and composition of the 2017-18 influenza vaccine. MMWR Morb Mortal Wkly Rep 66(25):668-676. https:// doi.org/10.15585/mmwr.mm6625a3

37. Hönemann M, Martin D, Pietsch C, Maier M, Bergs S, Bieck E et al (2019) Influenza B virus infections in Western Saxony, Germany in three consecutive seasons between 2015 and 2018: analysis of molecular and clinical features. Vaccine 37(43):6550-6557. https://doi.org/10.1016/j.vaccine.2019.08.027

38. Lugovtsev VY, Vodeiko GM, Strupczewski CM, Ye Z, Levandowski RA (2007) Generation of the influenza B viruses with improved growth phenotype by substitution of specific amino acids of hemagglutinin. Virology 365:315-323. https://doi.org/ 10.1016/j.virol.2007.04.006

39. Tramuto F, Orsi A, Maida CM, Costantino C, Trucchi C, Alicino $\mathrm{C}$ et al (2016) The molecular epidemiology and evolutionary dynamics of influenza B virus in two Italian regions during 2010-2015: the experience of Sicily and Liguria. Int J Mol Sci 17(4):549. https://doi.org/10.3390/ijms17040549

Publisher's Note Springer Nature remains neutral with regard to jurisdictional claims in published maps and institutional affiliations. 